Editorial

\title{
Longitudinal changes in cardiac biomarkers and outcome in heart failure: Sex-related differences
}

\author{
Alberto Aimo ${ }^{\mathrm{a}, \mathrm{b}, *}$ \\ a Scuola Superiore Sant'Anna, Pisa, Italy \\ b Fondazione Toscana Gabriele Monasterio, Pisa, Italy
}

Circulating biomarkers related to disease pathways involved in heart failure (HF) development and progression may be valuable tools for the diagnosis and management of HF [1,2]. Natriuretic peptides (NPs) are cardiac protective hormones released by cardiomyocytes in response to pressure or volume overload. Measurement of B-type NP (BNP) or $\mathrm{N}$-terminal pro-BNP (NT-proBNP) is recommended for risk stratification. The added value of serial measurements and the interpretation of changes in NPs over time are less well established. Furthermore, NP levels should be interpreted on the light of many factors, including sex [3] as well as age, kidney function, arrhythmias and obesity, among others. Other biomarkers can provide additional information to NPs. Many biomarkers have been evaluated, linked to specific mechanisms of neurohormonal activation, myocardial damage, cardiac remodelling, inflammation/oxidative stress and comorbidities. High-sensitivity troponins (hs-Tn) and soluble suppression of tumorigenesis-2 (sST2) are currently the most promising biomarkers as additive tools for risk stratification [4,5], but additional biomarkers have been proposed, such as C-reactive protein or several indicators of renal function [6].

A great research effort is underway to refine the use of biomarkers for outcome prediction in HF, with many studies on prospective cohorts or sub-analyses of randomized controlled trials [1,2]. In this issue of the Journal, Schreuder et al. evaluate the interplay between sex and the temporal patterns and prognostic impact of several biomarkers in patients with chronic HF [7]. The Authors evaluated 184 men and 66 women from the observational Bio-SHiFT study, all with $\mathrm{HF}$ and reduced ejection fraction (HFrEF; mean left ventricular ejection fraction [LVEF] 30\%). These patients underwent a blood sampling every 3 months with the assessment of 2 cardiac-specific biomarkers (NT-proBNP and hs-TnT), C-reactive protein (CRP), and 4 markers of renal function or tubular damage: creatinine, KIM1, cystatin $C$ and $\mathrm{N}$-Acetyl-beta-D-Glucosaminidase (NAG). Over a median follow-up of 2.2 years, 52 men and 14 women reached a composite endpoint of cardiac death, cardiac transplantation, left ventricular assist device implantation, and HF hospitalization. Patients experiencing the endpoint had higher levels of NT-proBNP, hs-TnT and CRP than those not experiencing the endpoint, with a progressive elevation approaching the endpoint, and consistently higher levels among women. Furthermore, these biomarkers tended to be more predictive of the endpoint in women than men. Conversely, markers of renal damage did not display sex-related differences in the changes over time or prognostic value. The Authors conclude that serial measurements of NT-proBNP, hs-TnT or CRP should be interpreted taking into consideration patient sex [7].

Point values of several biomarkers, first of all NPs and hs-TnT, are known to hold strong prognostic significance in $\operatorname{HFrEF}[3,8]$. Conversely, the need for repeated measurements of the same biomarkers and the interpretation of dynamic changes over time are less well established. This paper touches these important topics as well as the presence and meaning of sex-related differences in circulating biomarkers. Still, the analysis remains quite descriptive, without trying to search for possible causes of differences in circulating levels and prognostic value of biomarkers between women and men. Furthermore, the Authors did not go beyond a qualitative assessment of biomarker trends in men and women, and their finding of non-significant differences in the prognostic value of biomarkers between men and women could derive from the small number of patients (particularly women) and events. We may add that the Authors did not adjust for NT-proBNP or other outcome predictors (as done for example in a study on serial SST2 measurements) [9], did not explore the added value of repeated biomarker measures over point measures, or multiple biomarkers over a single biomarker such as NT-proBNP, either in the whole population or in men and women separately. Overall, the possibility to translate these findings in clinical practice is uncertain, and we agree with the Authors that "additional studies with larger sample sizes are warranted in order



* Corresponding author at: Institute of Life Sciences, Scuola Superiore Sant'Anna, Cardiology Division, Fondazione Toscana Gabriele Monasterio, Piazza Martiri della Libertà 33, 56124 Pisa, Italy.

URL's: a.aimo@santannapisa.it.
} 
to confirm these findings, while molecular biology studies are needed to further unravel underlying mechanisms" [7].

\section{Conflicts of interest}

None.

\section{References}

[1] S.L. Chow, A.S. Maisel, I. Anand, et al., Role of biomarkers for the prevention, assessment, and management of heart failure: a scientific statement from the American Heart Association, Circulation 135 (2017) e1054-e1091.

[2] V. Castiglione, A. Aimo, G. Vergaro, L. Saccaro, C. Passino, M. Emdin, Biomarkers for the diagnosis and management of heart failure, Heart Fail. Rev. (2021 Apr 14) https://doi.org/10.1007/s10741-021-10105-w (Epub ahead of print).
[3] L.B. Daniels, A.S. Maisel, Cardiovascular biomarkers and sex: the case for women, Nat Rev. Cardiol. 12 (2015) 588-596.

[4] A. Aimo, J.L. Januzzi Jr., G. Vergaro, et al., Prognostic value of high-sensitivity troponin $\mathrm{T}$ in chronic heart failure: an individual patient data meta-analysis, Circulation 137 (2018) 286-297.

[5] M. Emdin, A. Aimo, G. Vergaro, et al., sST2 predicts outcome in chronic heart failure beyond NT-proBNP and high-sensitivity troponin T, J. Am. Coll. Cardiol. 72 (2018) 2309-2320.

[6] A. Aimo, J.L. Januzzi Jr., G. Vergaro, et al., High-sensitivity troponin T, NT-proBNP and glomerular filtration rate: a multimarker strategy for risk stratification in chronic heart failure, Int. J. Cardiol. 277 (2019) 166-172.

[7] M.M. Schreuder, A. Schuurman, K.M. Akkerhuis, et al., Sex-specific temporal evolution of circulating biomarkers in patients with chronic heart failure with reduced ejection fraction, Int. J. Cardiol. (2021) (in press).

[8] R. Rørth, P.S. Jhund, M.B. Yilmaz, et al., Comparison of BNP and NT-proBNP in patients with heart failure and reduced ejection fraction, Circ. Heart Fail. 13 (2020) e006541.

[9] L.C. van Vark, I. Lesman-Leegte, S.J. Baart, et al., Prognostic value of serial ST2 measurements in patients with acute heart failure, J. Am. Coll. Cardiol. 70 (2017) 2378-2388. 